Jean-Michel Salaün*

\title{
Die drei Dimensionen des Dokuments und ihre Auswirkungen auf die Bibliotheks- und Informationswissenschaft
}

https://doi.org/10.1515/bfp-2018-0039

Zusammenfassung: Das Dokument wird in seinen drei Dimensionen analysiert: die Form, der Inhalt und die Funktion der Vermittlung. Das Web wird als die Erscheinungsform einer neuen dokumentarischen Ordnung verstanden, die geeigneter ist, den Anforderungen einer tiefgreifend gewandelten Welt $\mathrm{zu}$ entsprechen. Apple, Google, Facebook, jede der drei Firmen setzen jeweils auf eine der drei Dokumentdimensionen. Die Informationsarchitektur lässt neue professionelle Kompetenzen entstehen, die zur unumgänglichen Erneuerung der Informationswissenschaften beitragen könnten.

Schlüsselwörter: Dokument; Pédauque; Gütertypologie; Nicht-Rivalität von Information; Informationsarchitektur; Lesen; Text; Bibliothek als Medium; Umbruch der dokumentarischen Ordnung; Spätmoderne

\section{The three Dimensions of a Document and its Reflexion on Library and Information Science}

\begin{abstract}
The document is analyzed in its three dimensions: the form, the content, and the mediation. The Web is understood as the manifestation of a new document sphere, which is more able to satisfy the challenges of a world in profound change. Apple, Google, and Facebook exploit either of the three document dimensions. The discipline of IA „Information Architecture“ helps to develop new professional competencies which could contribute to an inevitable renewal of information sciences.
\end{abstract}

Keywords: Document; Pédauque; typology of goods; nonrivaltry of information; information architecture; reading; text; revolution of the documentary order; library as a medium; late modernity

\section{Die Bibliothek als Medium}

Die Bibliothek sammelt Dokumente, bewahrt sie auf und klassifiziert sie, um sie ihren Zielgruppen zur Verfügung zu stellen. Sie ist bei weitem das älteste aller Medien, das jedoch in neuerer Zeit durch die Digitalisierung erschüttert scheint. Bei der Digitalisierung handelt es sich nicht um die erste Hilfstechnologie im Bereich der Medien, die das bewirkt. Der Buchdruck mit seinen beweglichen Lettern hatte die Bibliothek bereits um die Funktion der Reproduktion und Zirkulation von Büchern gebracht, die früher durch die Buchkopier-Werkstätten zu ihren ureigensten Funktionen gezählt hatte.

Nach Experimenten mit mehreren amerikanischen Universitätsbibliotheken ${ }^{1}$ hatte Elsevier Mitte der 1990erJahre sein sehr profitables Lizenzmodell für elektronische wissenschaftliche Zeitschriften entwickelt, das seitdem von anderen großen Verlagshäusern nachgeahmt wird. Dadurch ist den Bibliotheken in dieser Sparte auch die angestammte Tätigkeit des Sammelns von Dokumenten verloren gegangen. Noch dramatischer ist es, dass Google - das Unternehmen mit einer der spektakulärsten Erfolgsgeschichten der Digitalwirtschaft - seine Suchmaschine auf einer riesigen Bibliothek von Kopien von Webseiten und anderen digitalen Dokumenten aufbaut. Google ist es auf diese Weise gelungen, ein Modell wirtschaftlich rentabel zu machen, das bis dahin auf einer nicht-kommerziellen Grundlage gearbeitet hatte.

Ist nun deshalb das älteste Medium, die traditionelle Bibliothek, endgültig obsolet geworden? Dies ist noch längst nicht ausgemacht. Die Bibliothek ist das Medium der langen Dauer, das sich seit seiner Gründung an die Entwicklung der Gesellschaften anpasst und stets ein moderates Tempo in die Überstürztheit der jeweiligen zeitgenössischen Medien bringt, die stürmisch und kurzlebig sind. Dennoch stellt die Digitalisierung die Bibliothek jetzt vor neue Herausforderungen.

1 Gusack and Lynch (1995). 


\section{Die drei Dimensionen des Dokuments}

Das Konzept des Dokuments ist uns heutzutage geläufig. Doch unter historischen Gesichtspunkten ist es eher neu, selbst wenn seine beiden Funktionen - übermitteln und nachweisen - alt sind. Seine Entstehung geht mit der Entwicklung der modernen Wissenschaft einher. Seine Blütezeit fällt mit der Entwicklung des systematischen Bibliotheksmodells zusammen, d.h. mit der Standardisierung der Methoden zur Katalogisierung und inhaltlichen Erschließung am Ende des 19. Jahrhunderts. ${ }^{2}$

Die Veränderungen des Dokuments bei seinem Wandel ins Digitale analysierend hat ein französisches Forscherteam, das unter dem Pseudonym Roger T. Pédauque ${ }^{3}$ bekannt geworden ist, vorgeschlagen, in den Dokumentbegriff mehr Klarheit zu bringen, indem man seine drei Dimensionen getrennt analysiert: die Form, der Text (oder Inhalt) und die Vermittlung (oder Übertragung). Die Kurzform für die drei Dimensionen sind die drei Substantive: „le vu“ (das Gesehene), „le lu“ (das Gelesene) und „le su“ (das Gewusste). ${ }^{4}$

\section{Die drei Dimensionen des Dokuments (am Beispiel des Buches)}

anthropologisch:

Form „le Vu“ (das Gesehene)

Dokument $=$ Träger + Inskription

intellektuell (neuro-anthropologisch):

Text „le Lu“ (das Gelesene)

Dokument $=$ Code + Repräsentation

sozial (sozio-anthropologisch):

Medium „le su“ (das Gewusste)

Dokument $=$ Gedächtnis + Transaktion
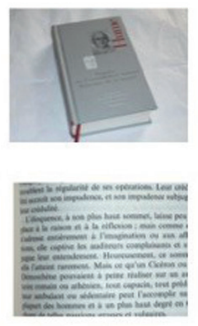

Abb. 1: Die drei Dimensionen des Dokuments (eigene Grafik)

Die erste Dimension des Dokuments, die der Form, ist anthropologischer Art. Sie betrifft die sinnliche Wahrnehmung des Objekts und seiner Eigenschaften. Sie wird dargestellt mit der Gleichung Dokument = Träger + Inskription .

2 Salaün (2014). Für eine Darstellung der Theorien des Dokuments siehe Buckland (2017).

3 Pédauque $(2003,2006)$

4 Salaün $(2012,2017)$
Die zweite Dimension ist intellektueller oder neuroanthropologischer Art. Hier handelt es sich um die Beziehung unseres Gehirns und seines Denkvermögens zum Inhalt des Dokuments, zum Text im weiteren Sinne. Diese Dimension wird dargestellt mit der Gleichung Dokument = Code + Repräsentation.

Die dritte Dimension ist eine soziale oder sozio-anthropologische. Es handelt sich um die Beziehung unseres Menschseins, unserer Position in einer Gesellschaft, zur Funktion des Dokuments in seiner Vermittlungsfähigkeit, unabhängig von seiner Form oder seinem Inhalt. Diese Dimension wird dargestellt mit der Gleichung Dokument = Gedächtnis + Transaktion.

\section{Die Notwendigkeit einer Lektüre-Vereinbarung}

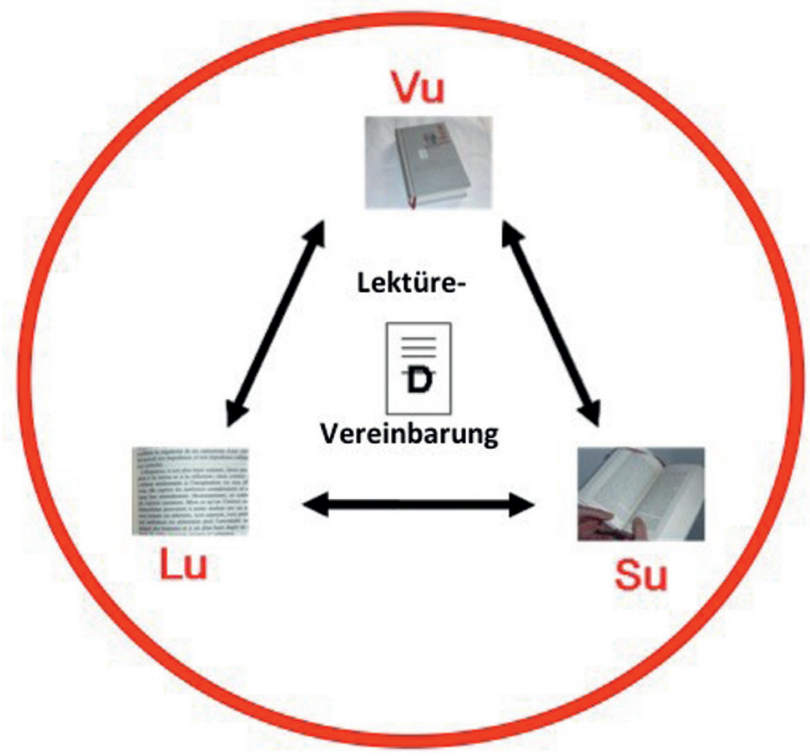

Abb. 2: Lektüre-Vereinbarung (eigene Grafik)

Nach dem von Pédauque vorgeschlagenen Konzept handelt es sich nur dann um ein Dokument, wenn seine drei Dimensionen in Beziehung gesetzt werden durch eine Lektüre-Vereinbarung, die die Produzenten oder Dokumentverantwortlichen mit seinen Nutzern, seinen Lesern, verbindet. Ein Pass, ein Buch, ein Testament, eine Zeitschrift, eine Rechnung, ein wissenschaftlicher Artikel, ein Film, eine Fernsehsendung usw. kommen nur dann in eine Produzent-Leser-Beziehung als Dokumente, wenn sie erstens in einem bestimmten Kontext als solche erkannt werden können ( $v u$ / das Gesehene); wenn man zweitens den Inhalt interpretieren kann (lu / das Gelesen) und wenn sie schließlich drittens eine Vermittlungsfunktion aufweisen (su / das Gewusste). Einen Pass werden Sie sofort erken- 
nen, wenn Sie ihn mit einem Blick zwischen verschiedenen gedruckten Papieren auf einem Tisch sehen. Wenn Sie ihn in die Hand nehmen und ihn lesen, werden Sie den Namen der Person, der er gehört und deren Herkunftsland nennen können. Und Sie wissen, dass diese Person den Pass benötigt, um zu reisen, um ihre Identität und Nationalität ausweisen zu können. Dies ist nur möglich, weil eine LektüreVereinbarung existiert, die Sie so sehr verinnerlicht haben, dass sie nun Teil Ihrer Denkreflexe geworden ist.

\section{Die Ökonomie des publizierten Dokuments}

Die Unterteilung des Dokuments entsprechend seiner drei Dimensionen eröffnet eine Anzahl von Analysepfaden. Wir werden hier nur einige davon aufzeigen. Diese Unterteilung ermöglicht es zum Beispiel, die Besonderheiten der Ökonomie des Dokuments besser zu verstehen.

Die Wirtschaftswissenschaftler betonen den Charakter der Nicht-Rivalität und der Nicht-Ausschließbarkeit von Information. Sie unterstreichen damit, dass eine Information von mehreren Empfängern geteilt werden kann, ohne dadurch verbraucht $\mathrm{zu}$ werden, im Gegensatz zu einem gewöhnlichen Wirtschaftsgut. Aber diese Eigenschaft betrifft nur die zweite Dimension des Dokuments, die des Textes, des Inhalts, die in dieser wirtschaftswissenschaftlichen Auffassung mit dem Sammelbegriff „Information“ gleichgesetzt wird.

Um überhaupt aufgenommen oder übertragen werden zu können, ist eine Information tatsächlich immer auf einem Träger eingeschrieben (inskribiert), an den sie angepasst oder durch den sie zerstört werden kann. Unter diesem Gesichtspunkt, nämlich dem der ersten Dimension des Dokuments, ist sie ein ganz normales rivalisierendes Gut. Viele Informationen sind im Laufe der Geschichte verschwunden, weil alle ihre Trägerobjekte zerstört worden sind.

Ferner gilt, dass wir während des Lesens eines Buches oder irgendeines Dokumentes bei der Aufnahme der Information unsere Aufmerksamkeit auf diese fokussieren. Wie Herbert Simon als erster gezeigt hat, ${ }^{5}$ ist die Aufmerksamkeit nicht teilbar. Besser noch: Je mehr Information im Überfluss vorhanden ist, desto knapper wird Aufmerksamkeit und desto größer wird der Wert der Aufmerksamkeit. Das Dokument ist gemäß seiner dritten Dimension - der der Übermittlung - also ebenfalls ein rivalisierendes Gut;

5 Simon (1971) $40 \mathrm{f}$. die Zeit und der Raum, die wir ihm widmen können, sind begrenzt.

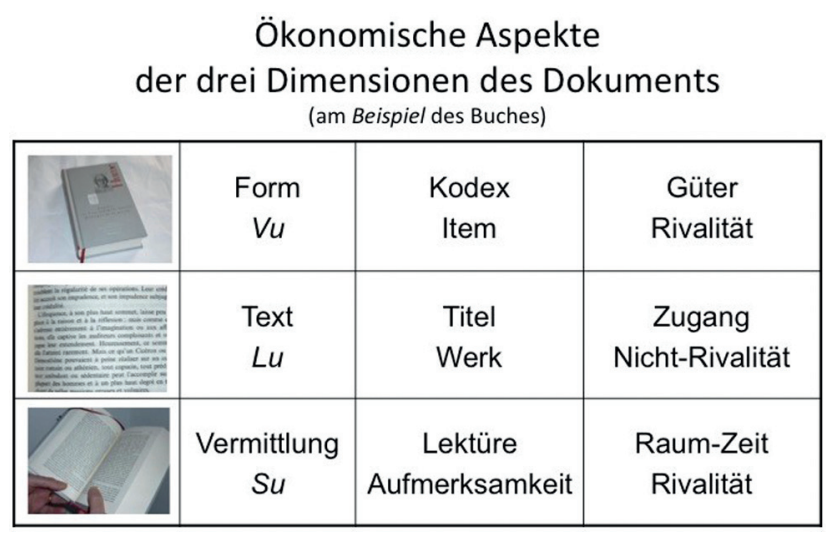

Abb. 3: Ökonomische Aspekte des Dokuments (eigene Grafik)

Infolgedessen haben sich für publizierte Dokumente drei und nur drei - Märkte eröffnet, die den drei Dimensionen des Dokuments entsprechen:

1. Verkauf von Gütern: das Verlagswesen. Man bindet die Information an den Träger, der dadurch gegen Entgelt getauscht werden kann.

2. Verkauf von Zugang: die Bibliothek. Man stellt die Information zur gemeinsamen Nutzung zur Verfügung. Man kann an die Gemeinschaft ein Zugangsticket verkaufen (Bibliotheksausweis).

3. Verkauf von Aufmerksamkeit: die Massenmedien [,le spectacle“ Anm. d. Übs.]. Man weckt die Aufmerksamkeit des „Empfängers“, die man an interessierte „Sender" verkauft.

Das Verlagswesen hat sich vor der Nicht-Rivalität der zweiten Dimension durch das Urheberrecht geschützt, das die Reproduktion von Texten untersagt. Demgegenüber hat die Bibliothek die Rivalität der ersten und dritten Dimension dadurch reduziert, dass sie zeitlich begrenzte Ausleihen oder Vorort-Nutzungen anbietet. Den Massenmedien ist es gelungen, ihren Markt zu vergrößern, indem sie die durch Inhalt gewonnene Aufmerksamkeit an interessierte Anzeigenkunden verkaufen.

Die Märkte sind im Laufe der letzten beiden Jahrhunderte zu eigenständigen Wirtschaftszweigen geworden, zunächst durch die Presse, dann durch Radio und Fernsehen und schließlich durch das Web, das die Vorteile dieser verschiedenen Märkte kombiniert und addiert. Darüber hinaus formt das Web die Gesamtheit der Medien integrierend zu Gedächtnisindustrien um, zu einer riesigen Bibliothek mit der Möglichkeit eines themenübergreifenden $\mathrm{Zu}$ gangs und einer dauerhaften Archivierung. 


\section{Der Umbruch in der dokumentarischen Ordnung}

Jede der Dokument-Dimensionen hat je nach Epoche mit unterschiedlicher Gewichtung ihre entsprechende Rolle gespielt. Mit der Erfindung des Buchdrucks ist die TextDimension vorherrschend geworden. Der Text, der in großer Anzahl und in identischer Form auf einem Träger reproduzierbar wurde, hat sich seitdem einerseits zu einem stabilen, trägerunabhängigen Element entwickelt und das Verlagswesen hat mit der massenhaften Verbreitung der Form seinen Aufschwung genommen. Wie es Elisabeth Eisenstein gezeigt hat, war es diese Eigenschaft des Textes, die die Entwicklung der Wissenschaften und das kritische Denken der Neuzeit in Europa begünstigt hat. ${ }^{6}$ Die Stabilität der zweiten Dimension (das Gelesene, d.h. die des Textes) bildet für die Moderne den Kern des Dokumentbegriffes.

Viele Fragen drehen sich heute um die Erschütterung dieser Dokumentstabilität durch die Entwicklungen des Digitalen. Das Digitale und insbesondere das Web lassen sich unter diesem Blickwinkel als Versuch deuten, das Konzept des Dokuments an die Spätmoderne anzupassen, um den Ausdruck von Hartmut Rosa aufzugreifen. ${ }^{7}$ Das Web wäre demnach, so wie der Buchdruck zu seiner Zeit, jetzt zum passenden Zeitpunkt entstanden, um an den Veränderungen unserer Wissensbeziehungen Teil zu haben, und diese zu relativieren und zu beschleunigen, zum Besseren wie zum Schlechteren.

Die hierarchische und systematische Dokumentorganisation, die aus dem 19. Jahrhundert stammt, dominierte im 20. Jahrhundert im Sinne einer Gesellschaft, die aus der industriellen und wissenschaftlichen Revolution hervorgegangen war. Das bibliografische Modell von FRBR, ${ }^{8}$ das das Werk, d.h. den Inhalt gegenüber seinem Informationsträger begünstigt, ist sicherlich davon die gelungenste Ausprägung aus bibliothekswissenschaftlicher Perspektive. Diese dokumentarische Ordnung stützt sich in einem Top-down-Verfahren auf den Sachverstand und die Weisheit der Gelehrten, wie dies Paul Otlet ${ }^{9}$ gezeigt hat.

Der Übergang zum Digitalen und ganz besonders der durch die Arbeit des W3C-Konsortiums bedingte Erfolg des Webs führte, bezogen auf das Dokument, zu einem radikalen Re-Engineering, das dies in seinen drei Dimensionen verändert hat. Mit der Digitalisierung hat die dritte Dimen-

6 Eisenstein (1979).

7 Rosa (2013).

8 Functional Requirements for Bibliographic Records.

9 Otlet (1934). sion, die Vermittlung, die Vorrangstellung gegenüber der zweiten Dimension übernommen.

Letztendlich stellt dieser Vorgang unsere DokumentWelt sogar auf den Kopf, in dem die Hierarchie der Konstruktion und der Selektion von Dokumenten umgekehrt wird. Die neue dokumentarische Ordnung stützt sich auf die Vielfalt der Internetnutzer, auf die „Weisheit der Vielen" mittels algorithmischer Berechnungen der Nutzerspuren (Google, Facebook) oder mittels Nutzer-Interaktionen (Wikipedia, Facebook). Dieses Re-Engineering des Dokumentes passt zu den aufkommenden Bedürfnissen und Werten einer multikulturellen, vergemeinschafteten und postmodernen Weltgesellschaft.

Die spektakulärsten Erscheinungsformen bei der Etablierung dieser neuen Dokument-Ordnung und ihrer Konkurrenz zur alten Ordnung sind die massive Nutzung von sozialen Netzwerken durch Politiker und durch unterschiedliche Aktivisten zur Verbreitung ihrer Meinungen und die irreführenden Werbekampagnen mithilfe gefälschter Nutzerkonten oder Hackerangriffen für wirtschaftliche oder politische Zwecke. Eine weitere durch diese neue Ordnung aufgeworfene Frage ist die der Überwachung und Kontrolle $\mathrm{zu}$ wirtschaftlichen, politischen oder polizeilichen Zwecken. Von dem Augenblick an, in dem die Vermittlungsfunktion die Vorherrschaft vor dem Text übernimmt, verlagert sich die Kontrollfunktion vom Inhalt auf die Nutzer. Eines der Symptome dieser Entwicklung sind die bei den Web-Firmen beliebten Design-Techniken auf der Basis von Nutzererfahrungen (UX = user experience design).

Diese schnellen Entwicklungen gehen einher mit neuartigen ethischen Problemen und diese neue Ordnung hat augenscheinlich ihren Regulierungsmodus noch nicht gefunden und destabilisiert die Vertreter der alten Ordnung, insbesondere die Journalisten und die akademische Welt.

\section{Die Strategien von Unternehmen im dokumentarischen Web}

Die Neuordnung der Dokumenten-Welt wird von der Dynamik privater Unternehmen getragen, die darin investiert haben. Deren Strategien können ebenfalls durch die dreidimensionale Dokument-Analyse erhellt werden. Durch Abschottungen oder Öffnungen bevorzugte man die eine oder andere Dimension des Dokuments.

Apple gründet seinen Reichtum als eine der ersten Firmen im digitalen Bereich auf sein Know-how im Bereich Design, also auf der Form, d. h. auf der ersten DokumentDimension. Apple verkauft uns für viel Geld Produkte, die 
unseren Zugang in sein vor-formatiertes Ökosystem einschließen und bietet diesen Zugang dann den Produzenten von Dokumenten und digitalen Anwendungen an.

Google bevorzugt die zweite Dimension, den Text. Die Basis-Kompetenzen von Google sind Computerlinguistik und statistische Lexikometrie. Das Geniale der Firma liegt darin, dass sie es verstanden hat, ihr Wissen zu nutzen, um einen janusköpfigen Markt (für Internetnutzer einerseits und für Anzeigenkunden andererseits) zu schaffen durch den Verkauf von Schlüsselwörtern, die der Wertigkeit der Suchanfragen von Internetnutzern zugeordnet werden.

Facebook, das seine Beherrschung des Social Graph als entscheidenden Wettbewerbsvorteil nutzt, um den Verkauf von Aufmerksamkeit zu Geld zu machen, polarisiert die Dokument-Ordnung in ihrer dritten Dimension, der Vermittlung.

Der sehr schnelle globale Aufstieg dieser drei Firmen und ihre Vereinnahmung der traditionellen dokumentarischen Vorgehensweisen belegen den Erfolg der neuen Dokument-Ordnung gegenüber der alten. Zahlreiche, insbesondere ethische und juristische Probleme bestehen weiterhin, so dass die Lage weit davon entfernt ist, stabil zu sein.

\section{Die Informationsarchitektur}

Angesichts dieser Umwälzungen müssen neue professionelle Kompetenzen entstehen und vielleicht sogar eine gänzlich neue Epistemologie. Der vielversprechendste Weg scheint mir hierbei der des Fachgebiets und der Community der „Informationsarchitektur“ zu sein, die vor einigen Jahren in den USA entstanden ist. Eines der wichtigsten Handbücher auf diesem Gebiet beschreibt sie wie folgt:

1. Die strukturelle Gestaltung geteilter Informationsräume

2. Die Synthese von Systemen der Organisation, Kategorisierung, Recherche und Navigation in digitalen, physikalischen und hybriden Ökosystemen

3. Die Theorie und Praxis der Gestaltung von Informationsprodukten und Informationserfahrungen zur Unterstützung der Nutzbarkeit, Auffindbarkeit und der Verständlichkeit von Information

4. Eine Fachdisziplin und eine wachsende Community von Praktikern, die die Prinzipien von Design und Architektur in die digitale Welt einbringen wollen.

Die englische Originalversion:

1. The structural design of shared information environments
2. The synthesis of organization, labeling, search, and navigation systems within digital, physical, and crosschannel ecosystems

3. The art and science of shaping information products and experiences to support usability and findability

4. An emerging discipline and community of practice focused on bringing principles of design and architecture to the digital landscape ${ }^{10}$

Vor kurzem hat sich die Thematik der Informationsarchitektur mit einer anderen wichtigen Bewegung verschränkt, die in den USA als „The Discipline of Organizing“111 entstanden ist. Zusammen mit einigen Kollegen haben wir versucht, deren Beiträge in Frankreich bekannt zu machen. ${ }^{12}$ Wir haben dafür ein Framework ${ }^{13}$ erarbeitet, das die Verbindung der drei wichtigsten Kompetenzbereiche betont: die Beherrschung der Informationsorganisation, die Beherrschung des Designs der Nutzererfahrung (user experience) und ein grundlegendes Verständnis der Konzepte digitaler Technologien. Die Verknüpfung dieser drei Kompetenzbereiche greift implizit die drei Dimensionen des Dokuments auf. Aber es bleibt noch viel zu tun, um das, was bis jetzt nur in Form einer Reihe praktischer Tätigkeiten besteht, in den Stand einer akademischen Disziplin zu erheben.

Die Konsolidierung der Bewegung, die sich um die Informationsarchitektur herum entwickelt hat, könnte dabei helfen, Theorie und Praxis der Informationswissenschaften zu erneuern. Unsere Disziplinen stellen sich nur mit Mühe den Herausforderungen des Digitalen, die jedoch sicher in Zukunft den Kernbereich unseres Gegenstands ausmachen wird. Die Informationswissenschaften wurden auf der Untersuchung einer Dokument-Welt aufgebaut, die heutzutage durch neue Praktiken erschüttert ist, denen die Informationsarchitektur aber Rechnung trägt und auf die sie schon einen fruchtbaren Einfluss nimmt. ${ }^{14}$

10 Rosenfeld et al. (2015) 24.

11 Glushko (2016).

12 Salaün et Habert (2015).

13 Habert et al. (2016), vgl. auch https://archinfo01.hypotheses.or $\mathrm{g} / 2018$.

14 Übersetzung des Artikels aus dem Französischen von Ulrike Becker und Hans-Christoph Hobohm. 


\section{Literaturverzeichnis}

Buckland, Michael (2017): Document theory. In: Encyclopedia of Knowledge Organization, ISKO. Online verfügbar unter http://w ww.isko.org/cyclo/document.

Eisenstein, Elizabeth L. (2009): The printing press as an agent of change: communications and cultural transformations in earlymodern Europe. Bde I und II. 14. Aufl. New York: Cambridge University Press. http://www.myilibrary.com?id=513315.

Glushko, Robert J. (Hrsg.) (2016): The Discipline of Organizing. Professional Edition. 4. Aufl. Sebastopol, CA: O'Reilly. Online verfügbar unter http://disciplineoforganizing.org/the-4th-edi tions/.

Gusack, Nancy; Lynch, Clifford A. (1995): The TULIP project. In: Library Hi Tec 13 (4), 7-24, https://doi.org/10.1108/eb047959.

Habert, Benoît; Salaün, JeanMichel; Magué, JeanPhilippe (2016): Référentiel de compétences en Architecture de l'information. Lyon: ENSSIB (Version 31.3.2016 verfügbar unter https://f-ori gin.hypotheses.org/wp-content/blogs.dir/888/files/2016/04/ Référentiel-Archinfo-2016.pdf zugegriffen am 8.2.2018.

Otlet, Paul (1934): Traité de documentation: le livre sur le livre, théorie et pratique. Bruxelles, Editions Mundaneum. Online verfügbar unter https://archive.org/stream/OtletTraitDocumentationUgen t\#page/n0/mode/1up.

Pédauque, Roger T. (2006): Le document à la lumière du numérique: forme, texte, medium: comprendre le rôle du document numérique dans l'émergence d'une nouvelle modernité. Caen: C \& F éd. https://cfeditions.com/pedauque.

Pédauque, Roger T. (2003): Document: Form, Sign and Medium, As Reformulated for Electronic Documents. Trad. by Lund, Niels Windfeld. Online verfügbar unter https://archivesic.ccsd.cnrs.f r/sic_00000594v1.

Rosa, Hartmut (2016): Beschleunigung und Entfremdung. Entwurf einer Kritischen Theorie spätmoderner Zeitlichkeit. Berlin: Suhrkamp.
Rosenfeld, Louis; Morville, Peter; Arango Jorge (2015): Information architecture for the World Wide Web. 4. Aufl. Beijing: O'Reilly.

Salaün, Jean-Michel (2015): Vu, lu, su: les architectes de l'information face à l'oligopole du Web. Paris: La Découverte. Als PDF verfügbar unter https://archinfocloud.ens-lyon.fr/public.php?s ervice $=$ files $\& \mathrm{t}=72 \mathrm{acf} 37169 \mathrm{ed} 1 \mathrm{~d} 3 \mathrm{f} 8118 \mathrm{c} 73 \mathrm{be} 50 \mathrm{ae} 8 \mathrm{a} 7$.

Salaün Jean-Michel (2014): Why The Document is Important ... And How it is Becoming Transformed. In: The Monist 97 (2) 187-99. Online unter https://doi.org/10.5840/monist201497213.

Salaün, Jean-Michel; Habert, Benoît (Hrsg.) (2015): Architecture de l'information - Méthodes, outils, enjeux. Louvain-la-Neuve: De Boeck.

Salaün, Jean-Michel (2017): Consolidation du nouvel ordre documentaire.

Postface à Vu, lu, su, cinq ans après. Als PDF verfügbar unter https:// archinfocloud.ens-lyon.fr/public. php? service $=$ files $\& t=c 11 b 5 f$ 949832873671790c6b88322b52.

Simon, Herbert A. (1971): Designing Organizations for an InformationRich World. In: Computers, Communication, and the Public Interest, hrsg. v. Martin Greenberger, Baltimore. MD: The Johns Hopkins Press, 37-72. http://digitalcollections.library.cmu.ed u/awweb/awarchive?type=file\&item $=33748$.

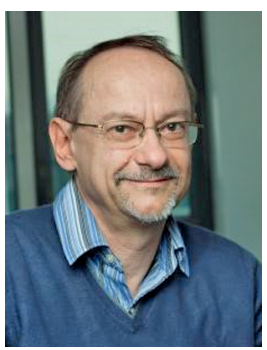

Prof. Dr. Jean-Michel Salaün Ecole Normale Supérieure de Lyon 114 Cours Tolstoi F-69100 Villeurbanne France salaunjm@gmail.com 